\title{
A result on precise asymptotics for largest eigenvalues of $\beta$ ensembles
}

Junshan $\mathrm{Xie}^{1 *}$ and Jing Zhao ${ }^{2}$

*Correspondence: xjsnwpu@163.com

${ }^{1}$ College of Mathematics and Information, Henan University, Kaifeng, 475000, P.R. China Full list of author information is available at the end of the article

\begin{abstract}
The paper focuses on the precise asymptotics of the largest eigenvalues of $\beta$-Hermite ensemble and $\beta$-Laguerre ensemble. In particular, we obtain a general law on the precise moment convergence rates for a type of weighted series constructed by the first-order conditional moment of the largest eigenvalues of $\beta$ ensembles. The results are motivated by the complete convergence for partial sums of independent random variables. The proofs depend on the small deviations for largest eigenvalue of $\beta$ ensembles and non-asymptotic tail probability inequalities of the general $\beta$ Tracy-Widom law.

MSC: $60 \mathrm{~F} 15 ; 60 \mathrm{G} 50 ; 60 \mathrm{G} 70$
\end{abstract}

Keywords: $\beta$ ensembles; largest eigenvalue; moment convergence rate; general $\beta$ Tracy-Widom law

\section{Introduction and main results}

In random matrix theory, $\beta$-Hermite ensemble and $\beta$-Laguerre ensemble introduced by Baker and Forrester [1] are two classical ensembles, and they have played an important role in lattice gas theory and statistical mechanics. The first one, denoted by $H_{\beta}$, is a probability measure related to a random point process on $\mathbb{R}$, whose joint probability density function has the form

$$
P\left(\lambda_{1}, \lambda_{2}, \ldots, \lambda_{n}\right)=\frac{1}{Z_{n, \beta}} \prod_{1 \leq j<k \leq n}\left|\lambda_{j}-\lambda_{k}\right|^{\beta} e^{-n \beta \sum_{k=1}^{n} \lambda_{k}^{2}}
$$

where $\lambda_{i} \in \mathbb{R}, Z_{n, \beta}$ is a normalizing constant, and for the parameter $\beta$ can be taken any $\beta>$ 0 and it can be interpreted as the inverse temperature of a Coulomb gas with logarithmic potential in lattice gas theory. In particular, when $\beta=1,2,4$, the joint densities are shared by the eigenvalues of Gaussian orthogonal ensemble $(G O E)$, Gaussian unitary ensemble $(G U E)$, and Gaussian symplectic ensemble (GSE), respectively.

For the Gaussian ensembles, because of the facts that there exist corresponding explicit random matrix models and that some powerful analytical methods are available in the derivation of their exact or asymptotical properties, lots of limiting spectral properties, including the behavior of the extremal eigenvalue, have been discussed in depth. As one of the major achievements in random matrix theory, it has been shown by Tracy and Widom [2-4] that the distribution of the largest eigenvalue of $G(O / U / S) E$, after being centered and scaled properly, converges to the Tracy-Widom type distribution, which is an important

\section{空 Springer}

○2014 Xie and Zhao; licensee Springer. This is an Open Access article distributed under the terms of the Creative Commons Attribution License (http://creativecommons.org/licenses/by/2.0), which permits unrestricted use, distribution, and reproduction in any medium, provided the original work is properly cited. 
probability distribution and has aroused great interest of the mathematicians and statisticians. According to the results of Tracy and Widom, for the largest eigenvalue $\lambda_{\max }(\mathcal{A})$ of the random matrix $\mathcal{A}$, whenever it comes from $\operatorname{GOE}(\beta=1)$, GUE $(\beta=2)$ or $\operatorname{GSE}(\beta=4)$, its distribution function

$$
F_{n, \beta}(x)=P\left(\lambda_{\max }(\mathcal{A}) \leq x\right), \quad \beta=1,2,4,
$$

satisfies the following Tracy-Widom limit law:

$$
\lim _{n \rightarrow \infty} F_{n, \beta}\left(\frac{x}{2 n^{\frac{2}{3}}}+1\right)=F_{\beta}(x), \quad \beta=1,2,4,
$$

which is given by

$$
\begin{aligned}
& F_{2}(x)=\exp \left(-\int_{x}^{\infty}(y-x) q^{2}(y) d y\right), \\
& F_{1}(x)=\exp \left(-\frac{1}{2} \int_{x}^{\infty} q(y) d y\right)\left(F_{2}(x)\right)^{\frac{1}{2}}, \\
& F_{4}\left(\frac{x}{\sqrt{2}}\right)=\cosh \left(\frac{1}{2} \int_{x}^{\infty} q(y) d y\right)\left(F_{2}(x)\right)^{\frac{1}{2}} .
\end{aligned}
$$

Here $q(x)$ is the unique solution to the Painlevé II equation $q^{\prime \prime}=x q+2 q^{3}$ with the asymptotics

$$
q(x) \sim \frac{1}{2} \pi^{-\frac{1}{2}} x^{-\frac{1}{4}} \exp \left(-\frac{2}{3} x^{\frac{3}{2}}\right) \quad \text { as } x \rightarrow \infty .
$$

As for the $\beta$-Hermite ensemble, where for $\beta>0$ can be taken any positive real value, a primary question is whether we can establish an internal relation with the random matrix model, whose eigenvalues obey the desired joint probability law. This problem was first posed by Dumitriu and Edelman [5], who successfully constructed a tridiagonal sparse random matrix model for $H_{\beta}$, and the eigenvalues of the matrix model obey the desired joint density function. We usually also call the corresponding matrix model as $H_{\beta}$ and denote the largest eigenvalue of the $\beta$-Hermite ensemble as $\lambda_{\max }\left(H_{\beta}\right)$. Making essential use of the tridiagonal matrix model, Ramírez et al. [6] proved that $\lambda_{\max }\left(H_{\beta}\right)$, after being centered and scaled, converges weakly to the general $\beta$ Tracy-Widom law $T W_{\beta}(\beta>0)$, that is,

$$
2 n^{\frac{2}{3}}\left(\lambda_{\max }\left(H_{\beta}\right)-1\right) \quad \Rightarrow \quad T W_{\beta}, \quad \beta>0 .
$$

They define the general $\beta$ Tracy-Widom law through a random variational principle:

$$
T W_{\beta} \stackrel{d}{=} \sup _{f \in L}\left\{\frac{2}{\sqrt{\beta}} \int_{0}^{\infty} f^{2}(x) d B(x)-\int_{0}^{\infty}\left[\left(f^{\prime}(x)\right)^{2}+x f^{2}(x)\right] d x\right\},
$$

where $x \mapsto B(x)$ is a standard Brownian motion, $L$ is the space of functions $f$ that vanish at the origin and satisfy $\int_{0}^{\infty} f^{2}(x) d x=1, \int_{0}^{\infty}\left[\left(f^{\prime}(x)\right)^{2}+x f^{2}(x)\right] d x<\infty$. 
Ramírez et al. [6] also obtained the tail properties of the distribution function of $T W_{\beta}$. Let $F_{\beta}(x)=P\left(T W_{\beta} \leq x\right)$ for any $\beta>0$ and large $a$, and we have

$$
F_{\beta}(-a)=\exp \left(-\frac{1}{24} \beta a^{3}(1+o(1))\right)
$$

and

$$
1-F_{\beta}(a)=\exp \left(-\frac{2}{3} \beta a^{\frac{3}{2}}(1+o(1))\right)
$$

In order to investigate the rates of the concentration for various eigenvalue statistics, some non-asymptotic results for certain random matrix ensembles have also been developed. Ledoux and Rider [7] presented some small deviation results related to $\lambda_{\max }\left(H_{\beta}\right)$, specifically, for all $n \geq 1,0<\varepsilon \leq 1$, and $\beta \geq 1$, we have

$$
P\left(\lambda_{\max }\left(H_{\beta}\right) \geq 1+\varepsilon\right) \leq C e^{-\frac{\beta}{C_{1}} n \varepsilon^{\frac{3}{2}}}
$$

and

$$
P\left(\lambda_{\max }\left(H_{\beta}\right) \leq 1-\varepsilon\right) \leq C e^{-\frac{\beta}{C_{1}} n^{2} \varepsilon^{\frac{3}{2}}},
$$

where $C$ and $C_{1}$ are positive constants.

In this paper, we are interested in the precise asymptotics of the largest eigenvalues of $\beta$ ensembles. Now we will give some attention to the precise asymptotics of random variables in classical probability theory. Let $\left\{X, X_{n} ; n \geq 1\right\}$ be a sequence of i.i.d. random variables, $S_{n}=\sum_{k=1}^{n} X_{k}$. Let $\varphi(x)$ and $f(x)$ be the positive functions defined on $[1,+\infty)$, which satisfy $\sum_{n=1}^{\infty} \varphi(n)=\infty, f(x) \uparrow \infty$ as $x \rightarrow \infty$. Since Hsu and Robbins [8] introduced the concept of complete convergence, some researchers discussed the convergence of the series $\sum_{n=1}^{\infty} \varphi(n) P\left(\left|S_{n}\right| \geq \varepsilon f(n)\right)$. Note that the sums tend to infinity as $\varepsilon \searrow 0$; it is interesting to find the precise convergence rates when this occurs. This amounts to finding the appropriate normalizations in terms of the functions of $\varepsilon$, which, multiplied by the series, has a non-trivial limit. The researchers call the discussion in this field 'precise asymptotics'. Later, some researchers extended the discussion to the cases that the series is constructed by conditional moment related to $S_{n}$, or the series is constructed by other variables, such as the associated counting process, empirical process, self-normalized sums. For more details on the topic of precise asymptotics of independent random variables, one can refer to Hsu and Robbins [8], Baum and Katz [9], Chow [10], Gut and Spǎtaru [11], Zhang et al. [12], Chen and Zhang [13] and Zang and Huang [14].

This paper is devoted to an extension of the precise asymptotics of i.i.d. to the eigenvalue variables of random matrices. $\mathrm{Su}$ [15] first presented some precise convergence asymptotic results on the largest eigenvalues of GUE and $L U E$, their results are in a sense similar to the precise asymptotics in the context of the complete convergence of independent random variables. Xie [16] further obtained the precise moment convergence rates of a type of weighted series constructed by the largest eigenvalues of the $\beta$ ensembles. The aim of the paper is to study the general law of the precise asymptotics of the moment convergence of the largest eigenvalues of the $\beta$ ensemble. 
Let $x_{+}=\max \{x, 0\}$ and $[x]$ be the integer part of real number $x$. The main results can be listed as follows.

Theorem 1.1 Assume that $g(x)$ is differentiable on the interval $[0,+\infty)$, which is nonnegative and strictly increasing to $\infty$, and the differentiable function $g^{\prime}(x)$ is non-negative. We further suppose that $g^{\prime}(x)$ is monotone, and if $g^{\prime}(x)$ is monotone non-decreasing, we have $\lim _{x \rightarrow \infty} \frac{g^{\prime}(x+1)}{g^{\prime}(x)}=1$. Then for any $s>0, \beta \geq 1$, we have

$$
\lim _{\varepsilon \searrow 0}(2 \varepsilon)^{\frac{1}{s}} \sum_{n=1}^{\infty} g^{\prime}(n) E\left\{n^{\frac{2}{3}}\left(\lambda_{\max }\left(H_{\beta}\right)-1\right)-\varepsilon g^{s}(n)\right\}_{+}=\frac{1}{2} \int_{0}^{\infty} x^{\frac{1}{s}}\left(1-F_{\beta}(x)\right) d x .
$$

Theorem 1.2 Under the assumptions of Theorem 1.1, we also have

$$
\lim _{\varepsilon \searrow 0} \frac{-1}{\log \varepsilon} \sum_{n=1}^{\infty} \frac{g^{\prime}(n)}{g(n)} E\left\{n^{\frac{2}{3}}\left(\lambda_{\max }\left(H_{\beta}\right)-1\right)-\frac{1}{2} \varepsilon g^{s}(n)\right\}_{+}=\frac{1}{2 s} \int_{0}^{\infty}\left(1-F_{\beta}(x)\right) d x .
$$

Remark 1.1 The assumptions on the function $g(x)$ in the above theorems are all rather mild conditions, it is easy to see that $g(x)=x^{a}, g(x)=(\log x)^{b}$ or $g(x)=(\log \log x)^{c}$ with some suitable conditions of $a>0, b>0$ or $c>0$ and some others all satisfy these assumptions. In particular, when $g(x)=x^{\frac{1}{3}}, s=2$, it can lead to a result which is consistent with that of Xie [16].

Remark 1.2 As it is difficult to get the explicit expression of $F_{\beta}(x)$, we do not present the exact values of the right hand of the above equations. However, the approximate values of them could be computed by Bornemann [17].

There is another probability measure, related to a random point process on $\mathbb{R}$ whose joint probability density function is

$$
P\left(\lambda_{1}^{\prime}, \lambda_{2}^{\prime}, \ldots, \lambda_{n}^{\prime}\right)=\frac{1}{Z_{n, \beta}^{\prime}} \prod_{1 \leq j<k \leq n}\left|\lambda_{j}^{\prime}-\lambda_{k}^{\prime}\right|^{\beta} \prod_{i=1}^{n} \lambda_{i}^{\left(\frac{\beta}{2}\right)(m-n+1)-1} e^{-\frac{n \beta}{2} \sum_{i=1}^{n} \lambda_{i}^{\prime},}
$$

where $\lambda_{i}^{\prime} \in \mathbb{R}^{+}, m>n-1$, and $\beta>0 ; Z_{n, \beta}^{\prime}$ is also a normalizing constant. It is usually called the $\beta$-Laguerre ensemble and denoted $L_{\beta}$ for short. Dumitriu and Edelman [5] also constructed a tridiagonal sparse random matrix model for $L_{\beta}$, and we usually also call the corresponding matrix model $L_{\beta}$ and denote the largest eigenvalue of the $\beta$-Hermite ensemble as $\lambda_{\max }\left(L_{\beta}\right)$. Ramírez et al. [6] also obtained, for $m+1>n \rightarrow \infty, \frac{m}{n} \rightarrow \gamma \geq 1$,

$$
\frac{m^{\frac{1}{6}} n^{\frac{7}{6}}}{(\sqrt{m}+\sqrt{n})^{\frac{4}{3}}}\left(\lambda_{\max }\left(L_{\beta}\right)-(1+\sqrt{\gamma})^{2}\right) \Rightarrow T W_{\beta} .
$$

At the same time, Ledoux and Rider [7] proved that for all $c_{1} n \leq m \leq c_{2} n$ with $c_{2} \geq c_{1} \geq 1$, $\beta \geq 1$, and $0<\varepsilon<1$, there exist $C>0, C_{1}>0$, and

$$
P\left(\lambda_{\max }\left(L_{\beta}\right) \geq\left(1+\sqrt{\frac{m}{n}}\right)^{2}(1+\varepsilon)\right) \leq C e^{-\frac{\beta}{C_{1}} \sqrt{m n \varepsilon} \frac{3}{2}\left(\frac{1}{\sqrt{\varepsilon}} \wedge\left(\frac{m}{n}\right)^{\frac{1}{4}}\right)} \leq C e^{-\frac{\beta}{C_{1}} n \varepsilon^{\frac{3}{2}}}
$$


and

$$
P\left(\lambda_{\max }\left(L_{\beta}\right) \leq\left(1+\sqrt{\frac{m}{n}}\right)^{2}(1-\varepsilon)\right) \leq C e^{-\frac{\beta}{C_{1}} m n \varepsilon^{3}\left(\frac{1}{\sqrt{\varepsilon}} \wedge\left(\frac{m}{n}\right)^{\frac{1}{2}}\right)} \leq C e^{-\frac{\beta}{C_{1}} n^{2} \varepsilon^{3}}
$$

With the help of the two important results, we could reach a similar result on the $\beta$ Laguerre ensemble.

Theorem 1.3 Under the assumptions of Theorem 1.1, when $m=[\gamma n], 1 \leq \gamma<\infty$ and $\beta \geq$ 1, for any $s>0$, we have

$$
\begin{aligned}
& \lim _{\varepsilon \searrow 0} \varepsilon^{\frac{1}{s}} \sum_{n=1}^{\infty} g^{\prime}(n) E\left\{\frac{m^{\frac{1}{6}} n^{\frac{7}{6}}}{(\sqrt{m}+\sqrt{n})^{\frac{4}{3}}}\left[\lambda_{\max }\left(L_{\beta}\right)-(1+\sqrt{\gamma})^{2}\right]-\varepsilon g^{s}(n)\right\}_{+} \\
& \quad=\int_{0}^{\infty} x^{\frac{1}{s}}\left(1-F_{\beta}(x)\right) d x .
\end{aligned}
$$

Theorem 1.4 Under the assumptions of Theorem 1.3, we have

$$
\begin{aligned}
& \lim _{\varepsilon \searrow 0} \frac{1}{-\log \varepsilon} \sum_{n=1}^{\infty} \frac{g^{\prime}(n)}{g(n)} E\left\{\frac{m^{\frac{1}{6}} n^{\frac{7}{6}}}{(\sqrt{m}+\sqrt{n})^{\frac{4}{3}}}\left[\lambda_{\max }\left(L_{\beta}\right)-(1+\sqrt{\gamma})^{2}\right]-\varepsilon g^{s}(n)\right\}_{+} \\
& \quad=\frac{1}{s} \int_{0}^{\infty}\left(1-F_{\beta}(x)\right) d x .
\end{aligned}
$$

The main proofs will be obtained in the next section, and they mainly depend on the non-asymptotic exponential inequalities about the general $\beta$ Tracy-Widom law, weak convergence, and small deviation inequalities about the largest eigenvalues of the $\beta$ ensembles. Throughout this paper, let $C$ denote an absolutely positive constant whose value can be different from one appearance to another.

\section{The proofs}

Proof of Theorem 1.1 Set $A(\varepsilon)=\left[g^{-1}\left(M \varepsilon^{-\frac{1}{s}}\right)\right]$, where $g^{-1}(x)$ is the inverse function of $g(x)$ and $M$ is an arbitrary positive real number. The proof of Theorem 1.1 relies on the following four propositions.

Proposition 2.1 Under the assumptions of Theorem 1.1, we have

$$
\lim _{\varepsilon \searrow 0} \varepsilon^{\frac{1}{s}} \sum_{n=1}^{\infty} g^{\prime}(n) E\left\{T W_{\beta}-2 \varepsilon g^{s}(n)\right\}_{+}=2^{-\frac{1}{s}} \int_{0}^{\infty} x^{\frac{1}{s}}\left(1-F_{\beta}(x)\right) d x .
$$

Proof Observing the fact that, for any random variable $\xi$ and $a \in R$,

$$
E \xi I_{\{\xi \geq a\}}=a P(\xi \geq a)+\int_{a}^{\infty} P(\xi \geq x) d x
$$

thus we have

$$
E\left\{T W_{\beta}-2 \varepsilon g^{s}(n)\right\}_{+}=\int_{2 \varepsilon g^{s}(n)}^{\infty} P\left(T W_{\beta} \geq t\right) d t
$$


If $g^{\prime}(x)$ is monotone non-increasing, by the assumption of Theorem 1.1, we can see that $g^{\prime}(x) \int_{2 \varepsilon g^{s}(x)}^{\infty} P\left(T W_{\beta} \geq t\right) d t$ is also non-increasing, thus

$$
\begin{aligned}
& \int_{1}^{\infty} g^{\prime}(y) \int_{2 \varepsilon g^{s}(y)}^{\infty} P\left(T W_{\beta} \geq t\right) d t d y \\
& \quad \leq \sum_{n=1}^{\infty} g^{\prime}(n) E\left\{T W_{\beta}-2 \varepsilon g^{s}(n)\right\}_{+} \\
& \quad \leq \int_{0}^{\infty} g^{\prime}(y) \int_{2 \varepsilon g^{s}(y)}^{\infty} P\left(T W_{\beta} \geq t\right) d t d y
\end{aligned}
$$

If $g^{\prime}(x)$ is monotone non-decreasing, by the assumption that $\lim _{n \rightarrow \infty} \frac{g^{\prime}(n+1)}{g^{\prime}(n)}=1$, for any $0<\delta_{0}<1$, there exists a sufficient large number $N=N\left(\delta_{0}\right)$, such that $\frac{g^{\prime}(n+1)}{g^{\prime}(n)}<1+\delta_{0}$ and $\frac{g^{\prime}(n+1)}{g^{\prime}(n)}>1-\delta_{0}$ for all $n \geq N$. Thus we can get

$$
\begin{aligned}
& \frac{1}{1+\delta_{0}} \int_{N}^{\infty} g^{\prime}(y) \int_{2 \varepsilon g^{s}(y)}^{\infty} P\left(T W_{\beta} \geq t\right) d t d y \\
& \quad \leq \sum_{n=N}^{\infty} g^{\prime}(n) E\left\{T W_{\beta}-2 \varepsilon g^{s}(n)\right\}_{+} \\
& \quad \leq \frac{1}{1-\delta_{0}} \int_{N-1}^{\infty} g^{\prime}(y) \int_{2 \varepsilon g^{s}(y)}^{\infty} P\left(T W_{\beta} \geq t\right) d t d y .
\end{aligned}
$$

Making a change of variables and performing integration by parts, for any $\theta \geq 0$, we can deduce that

$$
\begin{aligned}
& \lim _{\varepsilon \searrow 0} \varepsilon^{\frac{1}{s}} \int_{\theta}^{\infty} g^{\prime}(y) \int_{2 \varepsilon g^{s}(y)}^{\infty} P\left(T W_{\beta} \geq t\right) d t d y \\
& =\lim _{\varepsilon \searrow 0} \frac{1}{s} 2^{-\frac{1}{s}} \int_{2 \varepsilon g^{s}(\theta)}^{\infty} x^{\frac{1}{s}-1} \int_{x}^{\infty} P\left(T W_{\beta} \geq t\right) d t d x \\
& =\lim _{\varepsilon \searrow 0} \frac{1}{s} 2^{-\frac{1}{s}} \int_{2 \varepsilon g^{s}(\theta)}^{\infty} P\left(T W_{\beta} \geq t\right) \int_{2 \varepsilon g^{s}(\theta)}^{t} x^{\frac{1}{s}-1} d x d t \\
& =\lim _{\varepsilon \searrow 0} 2^{-\frac{1}{s}} \int_{2 \varepsilon g^{s}(\theta)}^{\infty}\left[t^{\frac{1}{s}}-(2 \varepsilon)^{\frac{1}{s}} g(\theta)\right]\left(1-F_{\beta}(t)\right) d t \\
& =2^{-\frac{1}{s}} \int_{0}^{\infty} t^{\frac{1}{s}}\left(1-F_{\beta}(t)\right) d t .
\end{aligned}
$$

By (2.3)-(2.5) and the fact that

$$
\lim _{\varepsilon \searrow 0} \varepsilon^{\frac{1}{s}} \sum_{n=1}^{\infty} g^{\prime}(n) E\left\{T W_{\beta}-2 \varepsilon g^{s}(n)\right\}_{+}=\lim _{\varepsilon \searrow 0} \varepsilon^{\frac{1}{s}} \sum_{n=N}^{\infty} g^{\prime}(n) E\left\{T W_{\beta}-2 \varepsilon g^{s}(n)\right\}_{+},
$$

letting $\delta_{0} \rightarrow 0$, we can complete the proof.

Proposition 2.2 Under the assumptions of Theorem 1.1, for any $M>0$, we have

$$
\lim _{\varepsilon \searrow 0} \varepsilon^{\frac{1}{s}} \sum_{n \leq A(\varepsilon)} g^{\prime}(n)\left|2 E\left\{n^{\frac{2}{3}}\left(\lambda_{\max }\left(H_{\beta}\right)-1\right)-\varepsilon g^{s}(n)\right\}_{+}-E\left\{T W_{\beta}-2 \varepsilon g^{s}(n)\right\}_{+}\right|=0 .
$$


Proof Using (1.2), we have

$$
\Delta_{n}:=\sup _{y \in R}\left|P\left(2 n^{\frac{2}{3}}\left(\lambda_{\max }\left(H_{\beta}\right)-1\right) \geq y\right)-P\left(T W_{\beta} \geq y\right)\right| \rightarrow 0 \quad \text { as } n \rightarrow \infty
$$

By (2.2), it is easy to see that

$$
\begin{aligned}
\mid 2 E & \left\{n^{\frac{2}{3}}\left(\lambda_{\max }\left(H_{\beta}\right)-1\right)-\varepsilon g^{s}(n)\right\}_{+}-E\left\{T W_{\beta}-2 \varepsilon g^{s}(n)\right\}_{+} \mid \\
& =\left|\int_{0}^{\infty} P\left(2 n^{\frac{2}{3}}\left[\lambda_{\max }\left(H_{\beta}\right)-1\right] \geq x+2 \varepsilon g^{s}(n)\right) d x-\int_{0}^{\infty} P\left(T W_{\beta} \geq x+2 \varepsilon g^{s}(n)\right) d x\right| \\
& \leq \int_{0}^{\infty}\left|P\left(2 n^{\frac{2}{3}}\left[\lambda_{\max }\left(H_{\beta}\right)-1\right] \geq x+2 \varepsilon g^{s}(n)\right)-P\left(T W_{\beta} \geq x+2 \varepsilon g^{s}(n)\right)\right| d x \\
& =\left(\Delta_{n 1}+\Delta_{n 2}\right),
\end{aligned}
$$

where

$$
\Delta_{n 1}=\int_{0}^{\Delta_{n}^{-\frac{1}{2}}}\left|P\left(2 n^{\frac{2}{3}}\left[\lambda_{\max }\left(H_{\beta}\right)-1\right] \geq x+2 \varepsilon g^{s}(n)\right)-P\left(T W_{\beta} \geq x+2 \varepsilon g^{s}(n)\right)\right| d x
$$

and

$$
\Delta_{n 2}=\int_{\Delta_{n}^{-\frac{1}{2}}}^{\infty}\left|P\left(2 n^{\frac{2}{3}}\left[\lambda_{\max }\left(H_{\beta}\right)-1\right] \geq x+2 \varepsilon g^{s}(n)\right)-P\left(T W_{\beta} \geq x+2 \varepsilon g^{s}(n)\right)\right| d x
$$

For the term $\Delta_{n 1}$, using the relation (2.6), we can get

$$
\Delta_{n 1} \leq \Delta_{n} \Delta_{n}^{-\frac{1}{2}}=\Delta_{n}^{\frac{1}{2}} \rightarrow 0 \quad \text { as } n \rightarrow \infty
$$

For the term $\Delta_{n 2}$, by (1.4) and (1.5), for large $n$, we obtain

$$
P\left(T W_{\beta} \geq x+2 \varepsilon g^{S}(n)\right) \leq C e^{-\frac{2}{3} \beta\left(x+2 \varepsilon g^{S}(n)\right)^{\frac{3}{2}}}
$$

and

$$
\begin{aligned}
P\left(2 n^{\frac{2}{3}}\left[\lambda_{\max }\left(H_{\beta}\right)-1\right] \geq x+2 n^{\frac{2}{3}} \varepsilon\right) & \leq C e^{-\frac{\beta n}{C_{1}}\left(\frac{1}{2} n^{-\frac{2}{3}} x+\varepsilon n^{-\frac{2}{3}} g^{s}(n)\right)^{\frac{3}{2}}} \\
& \leq C e^{-\frac{1}{C_{1}} \beta x^{\frac{3}{2}}} .
\end{aligned}
$$

Thus

$$
\Delta_{n 2} \leq C \int_{\Delta_{n}^{-\frac{1}{2}}}^{\infty} e^{-\frac{1}{C_{1}} \beta x^{\frac{3}{2}}} d x+C \int_{\Delta_{n}^{-\frac{1}{2}}}^{\infty} e^{-\frac{2}{3} \beta\left(x+2 \varepsilon g^{s}(n)\right)^{\frac{3}{2}}} d x \rightarrow 0 \quad \text { as } n \rightarrow \infty .
$$

Note that

$$
\varepsilon^{\frac{1}{s}} \sum_{n \leq A(\varepsilon)} g^{\prime}(n) \leq C \varepsilon^{\frac{1}{s}} \int_{0}^{A(\varepsilon)} g^{\prime}(x) d x=C \varepsilon^{\frac{1}{s}} g(A(\varepsilon))=C M<\infty,
$$


by the Toeplitz lemma (see Loève [18], p.250), we can find that

$$
\varepsilon^{\frac{1}{\bar{s}}} \sum_{n \leq A(\varepsilon)} g^{\prime}(n)\left(\Delta_{n 1}+\Delta_{n 2}\right) \rightarrow 0 \quad \text { as } \varepsilon \searrow 0 .
$$

Then the proof is completed.

Proposition 2.3 Under the assumptions of Theorem 1.1, for uniformly $\varepsilon>0$, we have

$$
\lim _{M \rightarrow \infty} \varepsilon^{\frac{1}{s}} \sum_{n>A(\varepsilon)} g^{\prime}(n) E\left\{T W_{\beta}-2 \varepsilon g^{s}(n)\right\}_{+}=0 .
$$

Proof Following the same line as of the proof of Proposition 2.1, we can deduce that

$$
\begin{aligned}
& \varepsilon^{\frac{1}{s}} \sum_{n>A(\varepsilon)} g^{\prime}(n) E\left\{T W_{\beta}-2 \varepsilon g^{s}(n)\right\}_{+} \\
& =\varepsilon^{\frac{1}{s}} \sum_{n>A(\varepsilon)} g^{\prime}(n) \int_{2 \varepsilon g^{s}(n)}^{\infty} P\left(T W_{\beta} \geq t\right) d t \\
& \leq C \varepsilon^{\frac{1}{s}} \int_{A(\varepsilon)}^{\infty} g^{\prime}(y) \int_{2 \varepsilon g^{s}(y)}^{\infty} P\left(T W_{\beta} \geq t\right) d t d y \\
& \leq C \int_{2 \varepsilon g^{s}(A(\varepsilon))}^{\infty} x^{\frac{1}{s}-1} \int_{x}^{\infty} P\left(T W_{\beta} \geq t\right) d t d x \\
& \leq C \int_{2 M^{s}}^{\infty} P\left(T W_{\beta} \geq t\right) \int_{2 M^{s}}^{t} x^{\frac{1}{s}-1} d x d t \\
& \leq C \int_{2 M^{s}}^{\infty} t^{\frac{1}{2}} e^{-\frac{2}{3} \beta t^{\frac{3}{2}}} d t \rightarrow 0 \text { as } M \rightarrow \infty,
\end{aligned}
$$

where (1.4) is used again in the last inequality. The proof is completed.

Proposition 2.4 Under the assumptions of Theorem 1.1, for uniformly $\varepsilon>0$, we have

$$
\lim _{M \rightarrow \infty} \sum_{n>A(\varepsilon)} g^{\prime}(n) E\left\{n^{\frac{2}{3}}\left(\lambda_{\max }\left(H_{\beta}\right)-1\right)-\varepsilon g^{S}(n)\right\}_{+}=0 .
$$

Proof It follows by the same argument as in Proposition 2.3 that

$$
\begin{aligned}
& \varepsilon^{\frac{1}{s}} \sum_{n>A(\varepsilon)} g^{\prime}(n) E\left\{n^{\frac{2}{3}}\left(\lambda_{\max }\left(H_{\beta}\right)-1\right)-\varepsilon g^{s}(n)\right\}_{+} \\
& \quad=\frac{1}{2} \varepsilon^{\frac{1}{s}} \sum_{n>A(\varepsilon)} g^{\prime}(n) \int_{2 \varepsilon g^{s}(n)}^{\infty} P\left\{2 n^{\frac{2}{3}}\left(\lambda_{\max }\left(H_{\beta}\right)-1\right) \geq t\right\} d t \\
& \quad \leq C \int_{2 M^{s}}^{\infty} P\left\{\lambda_{\max }\left(H_{\beta}\right) \geq 1+\frac{1}{2} n^{-\frac{2}{3}} t\right\} \int_{2 M^{s}}^{t} x^{\frac{1}{s}-1} d x d t \\
& \quad \leq C \int_{2 M^{s}}^{\infty} t^{\frac{1}{2}} e^{-\frac{\beta}{C_{1}} t^{\frac{3}{2}}} d t \rightarrow 0 \quad \text { as } M \rightarrow \infty,
\end{aligned}
$$

where the last inequality used the fact (1.5) again, and the proof is completed. 
Letting $\varepsilon \searrow 0$ and then $M \rightarrow \infty$, a combination of Propositions 2.1-2.4 can complete the proof of Theorem 1.1 immediately.

Proof of Theorem 1.2 The proof of Theorem 1.2 is analogous to that of Theorem 1.1. The only difference is that we will change the cut-off point $A(\varepsilon)=\left[g^{-1}\left(M \varepsilon^{-\frac{1}{s}}\right)\right]$ to a new one, $\hat{A}(\varepsilon)=\left[g^{-1}\left(\varepsilon^{-v}\right)\right], v>\frac{1}{s}$. The following four propositions corresponding to Propositions 2.12.4 are required, and the details are omitted.

Proposition 2.5 Under the assumptions of Theorem 1.2, we have

$$
\lim _{\varepsilon \searrow 0} \frac{-1}{\log \varepsilon} \sum_{n=1}^{\infty} \frac{g^{\prime}(n)}{g(n)} E\left\{T W_{\beta}-\varepsilon g^{s}(n)\right\}_{+}=\frac{1}{s} \int_{0}^{\infty}\left(1-F_{\beta}(x)\right) d x .
$$

Proposition 2.6 Under the assumptions of Theorem 1.2, we have

$$
\lim _{\varepsilon \searrow 0} \frac{-1}{\log \varepsilon} \sum_{n \leq \hat{A}(\varepsilon)} \frac{g^{\prime}(n)}{g(n)}\left|2 E\left\{n^{\frac{2}{3}}\left(\lambda_{\max }\left(H_{\beta}\right)-1\right)-\frac{1}{2} \varepsilon g^{s}(n)\right\}_{+}-E\left\{T W_{\beta}-\varepsilon g^{S}(n)\right\}_{+}\right|=0 .
$$

Proposition 2.7 Under the assumptions of Theorem 1.2, we have

$$
\lim _{\varepsilon \searrow 0} \frac{-1}{\log \varepsilon} \sum_{n>\hat{A}(\varepsilon)} \frac{g^{\prime}(n)}{g(n)} E\left\{T W_{\beta}-\varepsilon g^{s}(n)\right\}_{+}=0 .
$$

Proposition 2.8 Under the assumptions of Theorem 1.2, we have

$$
\lim _{\varepsilon \searrow 0} \frac{-1}{\log \varepsilon} \sum_{n>\hat{A}(\varepsilon)} \frac{g^{\prime}(n)}{g(n)} E\left\{n^{\frac{2}{3}}\left(\lambda_{\max }\left(H_{\beta}\right)-1\right)-\frac{1}{2} \varepsilon g^{S}(n)\right\}_{+}=0 .
$$

Proofs of Theorems 1.3 and 1.4 The proofs of Theorems 1.3 and 1.4 are essentially the same as those of Theorems 1.1 and 1.2, respectively, and the details of the proofs will not be presented.

\section{Competing interests \\ The authors declare that they have no competing interests.}

Authors' contributions

JX designed the work and drafted the manuscript, and JZ participated in the calculation of the work. All authors read and approved the final manuscript.

\section{Author details}

'College of Mathematics and Information, Henan University, Kaifeng, 475000, P.R. China. ${ }^{2}$ Department of Mathematics, Kaifeng Institute of Education, Kaifeng, 475000, P.R. China.

\section{Acknowledgements}

This work is supported by the National Natural Science Foundation of China (Nos. 11326173 and 11401169) and Foundation of Henan Educational Committee (No. 13A110087).

\section{Received: 30 April 2014 Accepted: 16 September 2014 Published: 16 Oct 2014}

\section{References}

1. Baker, T, Forrester, P: The Calogero-Sutherland model and generalized classical polynomials. Commun. Math. Phys. $188,175-216(1997)$

2. Tracy, C, Widom, H: Level-spacing distributions and the Airy kernel. Phys. Lett. B 305, 115-118 (1993) 
3. Tracy, C, Widom, H: Level-spacing distributions and the Airy kernel. Commun. Math. Phys. 159, 151-174 (1994)

4. Tracy, C, Widom, H: On orthogonal and symplectic matrix ensembles. Commun. Math. Phys. 177, 727-754 (1996)

5. Dumitriu, I, Edelman, A: Matrix models for beta ensembles. J. Math. Phys. 43, 5830-5847 (2002)

6. Ramírez, J, Rider, B, Virág, B: Beta ensembles, stochastic Airy spectrum and a diffusion. J. Am. Math. Soc. 24(4), 919-944 (2011)

7. Ledoux, M, Rider, B: Small deviations for beta ensembles. Electron. J. Probab. 15, 1319-1343 (2010)

8. Hsu, PL, Robbins, H: Complete convergence and the strong law of large number. Proc. Natl. Acad. Sci. USA 33(2), 25-31 (1947)

9. Baum, LE, Katz, M: Convergence rates in the law of large numbers. Trans. Am. Math. Soc. 120, 108-123 (1965)

10. Chow, YS: On the rates of moment convergence of sample sums and extremes. Bull. Inst. Math. Acad. Sin. 16, 177-201 (1988)

11. Gut, A, Spătaru, A: Precise asymptotics in the Baum-Katz and Davis law of large numbers. J. Math. Anal. Appl. 248 233-246 (2000)

12. Zhang, $Y$, Yang, $X Y$, Dong, ZS: A general law of precise asymptotics for the complete moment convergence. Chin. Ann. Math., Ser. B 30, 77-90 (2009)

13. Chen, Y, Zhang, L: Second moment convergence rates for uniform empirical processes. J. Inequal. Appl. 2010, Article ID $972324(2010)$

14. Zang, QP, Huang, W: A general law of moment convergence rates for uniform empirical process. Acta Math. Sin. Engl. Ser. 27(10), 1941-1948 (2011)

15. Su, ZG: Precise asymptotics for random matrices and random growth models. Acta Math. Sin. Engl. Ser. 24(6), 971-982 (2008)

16. Xie, JS: The moment convergence rates for largest eigenvalues of $\beta$ ensembles. Acta Math. Sin. Engl. Ser. 29(3), 477-488 (2013)

17. Bornemann, F: On the numerical evaluation of distributions in random matrix theory: a review with an invitation to experimental mathematics. Markov Process. Relat. Fields 16, 803-866 (2010)

18. Loève, M: Probability Theory, 4th edn. Springer, New York (1977)

10.1186/1029-242X-2014-408

Cite this article as: Xie and Zhao: A result on precise asymptotics for largest eigenvalues of $\beta$ ensembles. Journal of Inequalities and Applications 2014, 2014:408

\section{Submit your manuscript to a SpringerOpen ${ }^{\ominus}$ journal and benefit from:}

- Convenient online submission

- Rigorous peer review

Immediate publication on acceptance

- Open access: articles freely available online

- High visibility within the field

- Retaining the copyright to your article 